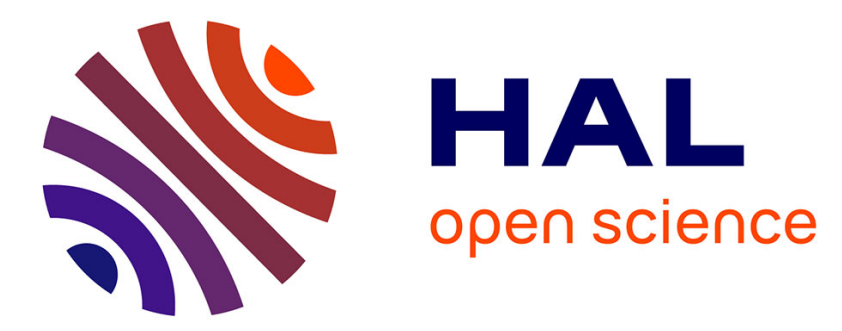

\title{
Parameterless discrete regularization on graphs for color image filtering
}

\author{
Olivier Lezoray, Sébastien Bougleux, Abderrahim Elmoataz
}

\section{To cite this version:}

Olivier Lezoray, Sébastien Bougleux, Abderrahim Elmoataz. Parameterless discrete regularization on graphs for color image filtering. 4th International Conference on Image Analysis and Recognition

(ICIAR 2007), Aug 2007, Montreal, Canada. pp.46-57, 10.1007/978-3-540-74260-9_5 . hal-00333522

\section{HAL Id: hal-00333522 \\ https://hal.science/hal-00333522}

Submitted on 23 Oct 2008

HAL is a multi-disciplinary open access archive for the deposit and dissemination of scientific research documents, whether they are published or not. The documents may come from teaching and research institutions in France or abroad, or from public or private research centers.
L'archive ouverte pluridisciplinaire HAL, est destinée au dépôt et à la diffusion de documents scientifiques de niveau recherche, publiés ou non, émanant des établissements d'enseignement et de recherche français ou étrangers, des laboratoires publics ou privés. 


\title{
Parameterless discrete regularization on graphs for color image filtering *
}

\author{
Olivier Lezoray $^{1}$, Sébastien Bougleux ${ }^{2}$, and Abderrahim Elmoataz ${ }^{1}$ \\ 1 Université de Caen, LUSAC EA 2607, Vision and Image Analysis Team, IUT SRC, \\ 120 Rue de l'exode, Saint-Lô, F-50000, France \\ olivier.lezoray@unicaen.fr, elmoataz@chbg.unicaen.fr \\ 2 ENSICAEN, GREYC, 6 Bd. Maréchal Juin, Caen, F-14050, France \\ sebastien.bougleux@greyc.ensicaen.fr
}

\begin{abstract}
A discrete regularization framework on graphs is proposed and studied for color image filtering purposes when images are represented by grid graphs. Image filtering is considered as a variational problem which consists in minimizing an appropriate energy function. In this paper, we propose a general discrete regularization framework defined on weighted graphs which can be seen as a discrete analogue of classical regularization theory. With this formulation, we propose a family of fast and simple anisotropic linear and nonlinear filters. The parameters of the proposed discrete regularization are estimated to have a parameterless filtering.
\end{abstract}

\section{Introduction}

Processing color images has became a crucial problem in the field of image processing. Numerous approaches can be found to process color images and among those, variational models have been extremely successful in a wide variety of computer vision problems such as image filtering and image segmentation. Variational formulations provide a framework that can handle such problems and provide algorithms for their solutions. Solutions of variational models can be obtained by minimizing appropriate energy functions, and this minimization is usually performed by designing continuous partial differential equations (PDEs). PDEs [1] are written in a continuous setting referring to images, and are discretized in order to have a numerical solution. One typical use of PDEs is image filtering and a lot of authors have proposed color image filtering with PDEs [2-5]. Discrete methods might be more suitable than PDEs in some cases since an image can be represented in a discrete setting by a grid graph. Discrete regularization on graphs has already been used for semi-supervised data classification [6]. Inspired by these works, we propose a regularization framework on weighted graphs of arbitrary topologies which defines a family of simple and fast anisotropic linear and nonlinear filters [7]. The discrete minimization problem is analogue to the continuous one and we propose to use such a discrete

\footnotetext{
* This research work was partially supported by the ANR foundation under grant ANR-06-MDCA-008-01/FOGRIMMI.
} 
regularization for parameterless filtering of color images. When $f^{0}$ denotes a degraded image of an image $f\left(f^{0}=f+\eta\right.$, where $\eta$ is additive noise of variance $\sigma^{2}$ ), the aim is to reconstruct $f$ from $f^{0}$ by regularization. The paper is organized as follows. In Section 2, after having recalled basic definitions on weighted graphs, we present differential geometry on graphs which is similar to the one proposed by Benssoussan [8] and Zhou [6]. In Section 3, we present a general framework for discrete regularization on graphs [7]. In section 4, we present a parameterless version of the proposed discrete regularization. In Section 5, we show how discrete regularization can be applied to color image filtering. Section 6 concludes.

\section{Differential geometry on graphs}

\subsection{Preliminaries on graphs}

A graph is a structure used to describe a set of objects and the pairwise relations between those objects (links between objects). The objects are called vertices (or nodes) and a link between two objects is called an edge. We provide some basic definitions on graph theory (further details can be found in [9]). A graph $\mathcal{G}$ is a couple $\mathcal{G}=(V, E)$ where $V$ is a finite set of vertices and $E$ is a set of edges included in a subset of $V \times V$. Two vertices $u$ and $v$ are adjacent if the edge $(u, v) \in E$. In the rest of this paper we only consider simple graphs which are always assumed to be connected, undirected with no self loops (see in $[9,6]$ for details on these notions). A graph as defined above is said to be weighted if it is associated with a weight function $w: E \rightarrow \mathbb{R}^{+}$satisfying $w(u, v)>0$ for $(u, v) \in E, w(u, v)=0$ for $(u, v) \notin E$ and $w(u, v)=w(v, u)$ for all edges in $E$ since we consider undirected graphs. The degree function $\delta: V \rightarrow \mathbb{R}^{+}$ of a vertex $v \in V$ is defined to be $\delta(v)=\sum_{u \sim v} w(u, v)$ where $u \sim v$ denotes all vertices $u$ connected to $v$ by an edge $(u, v) \in E$. Now we can define the space of functions on graphs. Let $\mathcal{H}(V)$ denote the Hilbert space of real-valued functions on vertices, in which each $f: V \rightarrow \mathbb{R}^{+}$assigns a real value $f(v)$ to each vertex $v$. The function space $\mathcal{H}(V)$ is endowed with the usual inner product $\langle f, g\rangle_{\mathcal{H}(V)}=\sum_{v \in V} f(v) g(v)$ where $f$ and $g$ are two functions in $\mathcal{H}(V)$. A function $f$ in $\mathcal{H}(V)$ can be thought as a column vector in $\mathbb{R}^{|V|}$. The norm of a function $f$ induced from the inner product is $\|f\|=\sqrt{\langle f, f\rangle_{\mathcal{H}(V)}}$. Similarly, one can define $\mathcal{H}(E)$ as the space of real-valued functions on edges, in which each $h: E \rightarrow \mathbb{R}^{+}$ assigns a real value to each edge $e \in E$. This function space is endowed with the usual inner product $\langle h, l\rangle_{\mathcal{H}(E)}=\sum_{(u, v) \in E} h(u, v) l(u, v)$ where $h, l: E \rightarrow \mathbb{R}^{+}$ denote two functions in $\mathcal{H}(E)$. In this paper, grid graphs are considered [10]. They correspond to the definition of digital images: vertices represent pixels and edges represent pixel adjacency relationship. Therefore, processing color images comes to processing grid graphs, the vertices models and edges weights of which depend on colorimetric properties of the image. 


\subsection{Gradient and divergence operators}

The difference operator $d: \mathcal{H}(V) \rightarrow \mathcal{H}(E)$ on $\mathcal{G}=(V, E)$ of a function $f \in \mathcal{H}(V)$ on an edge $(u, v)$ linking two vertices $u$ and $v$ is defined for all $(u, v) \in E$ as

$$
(d f)(u, v)=(d f)_{u v}=\sqrt{w(u, v)}(f(v)-f(u))
$$

The directional edge derivative of a function $f$ at vertex $v$ along the edge $e=$ $(u, v)$ is defined as $\partial_{v} f(u)=(d f)(u, v)$. This definition is consistent with the continuous definition of the derivative of a function, e.g., if $f(v)=f(u)$ then $\partial_{v} f(u)=0$. Moreover, one has $\partial_{v} f(u)=-\partial_{u} f(v)$ and $\partial_{u} f(u)=0$. Given a function $f \in \mathcal{H}(V)$ and a vertex $v$, the gradient of $f$ at vertex $v$ is the vector operator $\nabla: V \rightarrow \mathbb{R}^{N}$ defined by $\nabla f(v)=\nabla_{v} f=\left(\partial_{v} f(u):(u, v) \in E, u \sim v\right)^{T}$. Then, the norm $\|\nabla\|: \mathbb{R}^{N} \rightarrow \mathbb{R}^{+}$of the graph gradient $\nabla f$ at vertex $v$ or the local variation of $f$ at vertex $v$ is defined as:

$$
\left\|\nabla_{v} f\right\|=\sqrt{\sum_{u \sim v}\left(\partial_{v} f(u)\right)^{2}}=\sqrt{\sum_{u \sim v} w(u, v)(f(v)-f(u))^{2}}
$$

Let $\mathcal{R}$ denote a functional on $\mathcal{H}(V)$, for any $p \in[1,+\infty)$ which is defined by $\mathcal{R}_{p}(f)=\sum_{v \in V}\left\|\nabla_{v} f\right\|^{p}$. This functional $\mathcal{R}_{p}$ can be seen as the measure of the smoothness of $f$ since it is the sum of the local variations at each vertex. The graph divergence operator is the operator div $: \mathcal{H}(E) \rightarrow \mathcal{H}(V)$ which satisfies $\langle d f, h\rangle_{\mathcal{H}(E)}=\langle f,-\operatorname{div}(h)\rangle_{\mathcal{H}(V)}$ with $f \in \mathcal{H}(V)$ and $h \in \mathcal{H}(E)$. The operator -div is therefore the adjoint operator $d^{*}$ of the difference operator $d$. From the definition of the inner products in $\mathcal{H}(V)$ and $\mathcal{H}(E)$ and Equation (1), one can prove that the graph divergence of a function $h \in \mathcal{H}(E)$ at a vertex $v$ can be expressed as

$$
\left(d^{*} h\right)(v)=(-\operatorname{div}(h))(v)=\sum_{u \sim v} \sqrt{w(v, u)}(h(u, v)-h(v, u))
$$

The divergence operator measures the net outflow of function $h \in \mathcal{H}(E)$ at each vertex $v$ of $\mathcal{G}$.

\section{3 p-Laplace operator}

The graph p-Laplacian is the operator $\Delta_{p}: \mathcal{H}(V) \rightarrow \mathcal{H}(V)$, with $p \in[1,+\infty)$, defined as

$$
\Delta_{p} f=-\operatorname{div}\left(\|\nabla f\|^{p-2} d f\right)=d^{*}\left(\|\nabla f\|^{p-2} d f\right)
$$

Substituting (1) and (3) into the definition (4) of $\Delta_{p} f$, we obtain

$$
\left(\Delta_{p} f\right)(v)=\sum_{u \sim v} \gamma(u, v)(f(v)-f(u))
$$

where $\gamma(u, v)$ is the function defined by

$$
\gamma(u, v)=w(u, v)\left(\|\nabla f(v)\|^{p-2}+\|\nabla f(u)\|^{p-2}\right)
$$


which generalizes the classical graph Laplacian and curvature. Indeed, the classical graph Laplacian is linear the operator $\Delta: \mathcal{H}(V) \rightarrow \mathcal{H}(V)$ defined as $\Delta f=-\operatorname{div}(d f)=d^{*}(d f)$ and the classical graph curvature is the nonlinear operator $\kappa: \mathcal{H}(V) \rightarrow \mathcal{H}(V)$ defined as $\kappa f=-\operatorname{div}\left(\frac{d f}{\|\nabla f\|}\right)=d^{*}\left(\frac{d f}{\|\nabla f\|}\right)$. Clearly, one has $\Delta_{1}=\kappa$ and $\Delta_{2}=\Delta$. In general $\Delta_{p}$ is nonlinear (except in the case of $p=2)$ and it is positive semi-definite. One can then prove that $\left\langle f, \Delta_{p} f\right\rangle_{\mathcal{H}(V)}=\mathcal{R}_{p}(f)=\sum_{v \in V}\left\|\nabla_{v} f\right\|^{p} \geq 0$, which implies that

$$
\Delta_{p} f=\frac{\partial \mathcal{R}_{p}(f)}{\partial f}
$$

Practically, to avoid having a zero denominator to compute the curvature (i.e. $p=1$ ), the graph gradient $\left\|\nabla_{v} f\right\|$ has to be replaced by its regularized version: $\left\|\nabla_{v} f\right\|_{\beta}=\sqrt{\beta^{2}+\left\|\nabla_{v} f\right\|^{2}}$ where $\beta>0$ is a small positive parameter called the regularization parameter [10]. For the sake of clarity, when it is not necessary, we keep the notation $\left\|\nabla_{v} f\right\|$ instead of $\left\|\nabla_{v} f\right\|_{\beta}$ in the rest of the paper.

\section{Discrete regularization on graphs}

In this section, we propose a general framework to regularize color images represented by grid graphs. For the sake of clarity we present this framework for scalar images but the principle is the same for color images (see next section). Given a graph $\mathcal{G}=(V, E)$ associated with a weighting function $w: E \rightarrow \mathbb{R}^{+}$, we want to perform the discrete regularization of a function $f^{0} \in \mathcal{H}(V)$ (i.e. the initial image) using the p-Laplacian. It consists in seeking for a function $f^{*}$ which is smooth and simultaneously close to the function $f^{0}$. This comes to consider general variational problems on graphs. Given a function $f^{0} \in \mathcal{H}(V)$, the goal is to find a function $f^{*} \in \mathcal{H}(V)$ which is not only smooth enough on $\mathcal{G}$ but also close enough to the given function $f$. This can be formalized by minimizing a weighted sum of two energy terms:

$$
f^{*}=\min _{f \in \mathcal{H}(V)}\left\{E_{p}=\mathcal{R}_{p}(f)+\lambda\left\|f-f^{0}\right\|^{2}=\sum_{v \in V}\left\|\nabla_{v} f\right\|^{p}+\lambda \sum_{v \in V}\left\|f-f^{0}\right\|^{2}\right\}
$$

The first term is the smoothness term or regularizer, which requires $f$ not to change too much between closely related objects. The second term is the fitting term, which says that $f$ should not be far away from $f^{0}$. The parameter $\lambda \geq 0$ is a fidelity parameter called the Lagrange multiplier which specifies the trade-off between the two competing terms. Both terms of the energy $E_{p}$ are strictly convex functions of $f[10,11]$, therefore, by standard arguments in convex analysis, this optimization problem has a unique solution for $p=1$ or $p=2$ which satisfies $\left.\frac{\partial E_{p}}{\partial f}\right|_{v}=0, \forall v \in V$. Using the property (7) of the pLaplacian to compute the derivative of the first term in $E_{p}$, the above mentioned 
problem can be rewritten as follows:

$$
\left(\Delta_{p} f^{*}\right)(v)+2 \lambda\left(f^{*}(v)-f^{0}(v)\right)=0, \forall v \in V
$$

The solution $f^{*}$ of (8) is also the solution of (9). Substituting the expression of the p-Laplacian into (9), we obtain:

$$
\left(2 \lambda+\sum_{u \sim v} \gamma_{u v}\right) f^{*}(v)-\sum_{u \sim v} \gamma(u, v) f^{*}(u)=2 \lambda f^{0}(v), \quad \forall v \in V .
$$

Among the existing methods which can be used to solve (10), we use the Gauss-Jacobi iterative algorithm. In this paper, we consider only the case of $p=1$ based on the nonlinear curvature operator $\kappa$. Let $t$ be the iteration step, and let $f^{(t)}$ be the solution of (10) at the step $t$. The initial function $f^{(0)}$ can be initialized to $f^{0}$. The corresponding linearized Gauss-Jacobi algorithm is given by:

$$
\left\{\begin{array}{l}
f^{(0)}=f^{0} \\
\gamma^{(t+1)}(u, v)=w(u, v)\left(\frac{1}{\left\|\nabla f^{(t+1)}(v)\right\|_{\beta}}+\frac{1}{\left\|\nabla f^{(t+1)}(u)\right\|_{\beta}}\right), \forall(u, v) \in E, \\
f^{(t+1)}(v)=\frac{2 \lambda}{2 \lambda+\sum_{u \sim v} \gamma^{t}(u, v)} f^{0}(v)+\frac{\sum_{u \sim v} \gamma^{t}(u, v) f^{t}(u)}{2 \lambda+\sum_{u \sim v} \gamma^{t}(u, v)}, \quad \forall v \in V,
\end{array}\right.
$$

where $\gamma^{(t)}$ is the function $\gamma(u, v)$ at the step $t$. One can note that the value of $f(v)$ for a given iteration $(t+1)$ depends on two quantities: the original value of $f$ at $v$ (i.e. $f^{0}(v)$ ) and the values for iteration $t$ in the neighborhood of $v$. Coefficients are associated to those quantities which depend on the sum of weighted local variations. The obtained filtering is a low pass filter where the coefficients are adaptively updated for each iteration in addition of updating the function $f$. It is worth to note the connection between the proposed filter and the TV digital filter [10] (TV $+L^{2}$ on grid graphs). Indeed, with $p=1$, if $\forall(u, v) \in E, w(u, v)=1$ i.e. the edges have all the same weights, one recovers exactly the same iterative filtering performed on a regular grid represented by a graph.

\section{Parameterless discrete regularization}

In this Section, we show how the proposed discrete regularization can be parameterless for the purpose of filtering color images. Indeed, several parameters act upon the proposed discrete regularization: the representation of color vectors associated to the vertices $(f(v), \forall v \in V)$, the weights associated to the edges $(w(u, v), \forall(u, v) \in E)$, the regularization constants $(\lambda$, but also $\beta)$, and the number of iterations involved in algorithm (11). 


\subsection{The case of color images}

For the case of color images, we define $f \in \mathcal{H}(V), f: V \rightarrow \mathbb{R}^{3}$ which associates a red-green-blue color vector to each vertex. To perform the regularization on a grid graph representing a color image, an iteration of (11) is considered. Since a color image is composed of three channels, three independent regularization processes are considered. To take the coupling between vector channels into account, the component-wise regularizations do not have to use different local geometries (the p-Laplacian being different for each channel with $p=1$ ) but a vector one. Therefore, the p-Laplace operator is considered as being the same for the three channel regularizations (channel coupling) and is defined by $\gamma(u, v)=w(u, v)\left(\|\nabla f(v)\|_{3 D}^{p-2}+\|\nabla f(u)\|_{3 D}^{p-2}\right)$. The norm of a color vector is defined as its multi-dimensional Euclidean norm and remains the same whatever the color channel under consideration is. This is required to have a global vector geometry $\|\nabla f(v)\|_{3 D}=\sqrt{\sum_{i=1}^{3}\left\|\nabla f_{i}(v)\right\|^{2}}$. Another model (ChromaticityBrightness denoted as CB) can be used to represent color images. It decomposes a RGB color vector $f(v)$ into two components: the brightness component $b(v)=\|f(v)\|$ and the chromaticity component $c(v)=f(v) / b(v)$. The discrete regularization is then performed separately on the scalar brightness component and on the vectorial chromaticity component (this involves a supplementary step of normalization to ensure that the chromaticity remains on the unit sphere, see [12] for further details).

\subsection{Edge weights}

We can associate a weight function defined for each edge of a graph. This weight function determines the type of regularization induced by the functional $\mathcal{R}_{p}(f)$. Weights are positive and symmetric and enable to quantify the proximity between two vertices based on some features. Therefore, similarities between vertices are obtained by comparing their features which generally depend on the function $f$ and the set $V$. A feature vector $F_{f}(v) \in \mathbb{R}^{q}$ is assigned to every vertex $v \in V$, with $q \in \mathbb{N}^{+}$. This feature vector can incorporate several image features such as color and texture. The general formulation of a weight function can be defined as $w(u, v)=g\left(F_{f}(u), F_{f}(v)\right), \forall(u, v) \in E$. We consider three different weight functions. $g_{1}\left(F_{f}(u), F_{f}(v)\right)=\frac{1}{\varepsilon+\left\|F_{f}(u)-F_{f}(v)\right\|}$ with $F_{f}(u)=f(u) \cdot g_{2}\left(F_{f}(u), F_{f}(v)\right)=\exp \left(-\frac{\left\|F_{f}(u)-F_{f}(v)\right\|^{2}}{\sigma^{2}}\right)$ with $F_{f}(u)=f(u)$. $g_{3}\left(F_{f}(u), F_{f}(v)\right)=\exp \left(-\frac{\left\|F_{f}(u)-F_{f}(v)\right\|^{2}}{\sigma^{2}}\right) \exp \left(-\frac{\|u-v\|^{2}}{2 \sigma_{d}^{2}}\right)$ with $F_{f}(u)=[f(v)$ : $\left.v \in W_{u}^{r}\right]^{T} . \sigma_{d}$ controls the spatial decay and $W_{u}^{r}$ denotes the set of vertices which can be reached from $u$ in $r$ walks (for grid graphs, $W_{u}^{r}$ is a window of size $2 \times r+1$ centered on $u$ ). This last weight function $g_{3}$ was proposed by BUADES for a nonlocal means filter[13]. We use it to define a nonlocal discrete regularization on graphs. All these weight functions involve parameters. For $g_{1}$, the parameter $\varepsilon$ is needed to avoid a zero denominator and it can be 
fixed with a very small value $\left(10^{-4}\right.$ in our experimentations). For $g_{2}$ and $g_{3}$, the parameter $\sigma$ is usually fixed a priori in literature. To have parameterless weight functions, a measure of dispersion around vertices can be used to estimate this parameter. We propose to estimate $\sigma$ for two nodes $u$ and $v$ as the product $\sigma_{u} \sigma_{v}$ of the standard deviation estimated around each vertex. Therefore,

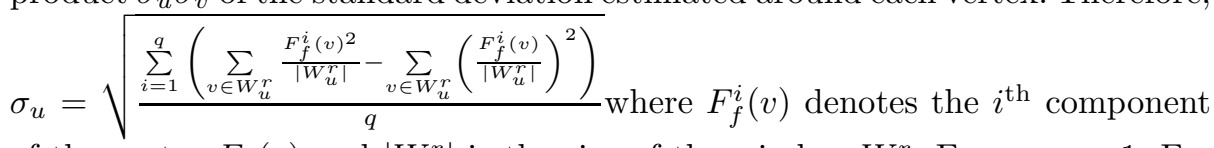
of the vector $F_{f}(v)$ and $\left|W_{u}^{r}\right|$ is the size of the window $W_{u}^{r}$. For $g_{2}, r=1$. For computational reasons, $\sigma_{u}$ is estimated only once for each vertex on the original image $f^{0}$. The proposed weight functions are now parameterless but their choice depends on the application (see next Section).

\subsection{Regularization constants}

The regularization constant $\lambda$ determines the trade-off between the smoothness of the regularized image and the closeness of the regularized image to the original image. It plays an important role in the regularization and should be estimated along the iterations rather than fixed a priori since it depends on the noise level. A way to estimate $\lambda$, it to consider the equivalent constrained minimization problem[14] of (8) formulated as: $\min _{f} \sum_{v \in V}\left\|\nabla_{v} f\right\|^{p}$ subject to $\frac{1}{|V|} \sum_{v \in V}\left\|f-f^{0}\right\|^{2}=$ $\sigma^{2}$. Then, using (7) and (5), one obtains, at a given iteration $t$, an estimation of $\lambda[10]: \lambda_{t}=\frac{1}{\bar{\sigma}_{t}^{2}} \frac{1}{|V|} \sum_{v \in V}\left(\left(f^{t}(v)-f^{0}(v)\right) \sum_{u \sim v} \gamma_{u v}\left(f^{t}(v)-f^{t}(u)\right)\right)$.For $t=0$, we fix $\lambda_{0}=\frac{1}{\bar{\sigma}_{t}^{2}}$ where $\bar{\sigma}_{t}^{2}$ denotes the variance of the noise estimated on the whole image $f^{t}$. With $p=1$, the regularization parameter $\beta$ (see Section 2.3) is needed for numerical stabilities but this leads to an approximation of the solution of (8). As stated in [10], the performance of the regularization is insensitive to $\beta$ as long as it is kept small. The use of $\beta$ is needed to reduce degeneracies in flat regions where $\left\|\nabla_{v} f\right\| \approx 0$ and is a commonly used technique [2]. Therefore, we fix $\beta^{2}=\frac{1}{\bar{\sigma}_{0}^{2}}$.

\subsection{Termination criterion}

To have a complete parameterless algorithm, the ideal number of iterations of (11) should by dynamically determined. To that aim, we use a termination criterion defined as $\frac{\left\|f^{t}(v)-f^{0}(v)\right\|}{\left\|f^{t}(v)\right\|}<\varepsilon$ ( $\varepsilon$ value is the same as in subsection 4.2$)$. This enables to automatically determine the stopping time of (11) when few modifications occur on $f^{t}$.

\section{Applications}

In this section, we show how the proposed parameterless discrete regularization can be used to perform color image filtering. For a more complete evaluation of 
the discrete regularization without automatic estimation of the parameters as defined in Section 4, one can refer to [7]. Images are represented by grid graphs (one vertex per pixel) with 8-neighborhood connectivity (filter window of size $3 \times 3)$. First, we consider an image (Figure 1(a)) corrupted by impulse noie (corruption of $15 \%$, Figure 1(b)) or Gaussian noise $(\sigma=15$, Figure 1(c)). For impulse noise cancelation, the weight function $g_{1}$ is considered. One can see on Figure 1(d) the behavior of the proposed parameterless discrete regularization. For Gaussian noise cancelation, the weight function $g_{2}$ is considered. Figure 1(e) and Figure 1(f) present filtering results respectively operating on the $R G B$ image or its representation in Chromaticity-Brigthness. The noise is suppressed and the use of Chromaticity-Brigthness features enables a better restoration of the image (see the background in Figure 1(f)). This illustrates that the parameterless discrete regularization performs well for color image filtering and it can be adapted to different types of noise by chosing an approriate weight function and color vector representation. We illustrate this last remark on a real noisy image (Figure 2(a)) captured by a digital camera at very high shutter speed using a high film ISO degree. Parameterless discrete regularization is performed with the following configurations; Figure 2(b) with $g_{1}$, Figure 2(c) with $g_{2}$, Figure 2(d) with $g_{3}(r=1)$ and Figure 2(d) with $g_{3}(r=1)$ on Chromaticity-Brigthness features. When $g_{3}$ is used, a 24-neighborhood connectivity is considered (filter window of size $5 \times 5$ ). Indeed, the feature vector associated to each vertex is defined over a 8-neighborhood $(r=1)$ and the filter window has to be larger than the size of the feature vector (see in [15] for more details). The weight function $g_{2}$ provides in general better results than $g_{1}$ (except for impulse noise). Moreover, the use of weight function $g_{3}$, which is nonlocal[13], provides better results than the same norm in a fully local version i.e. with weight function $g_{2}$ (compare Figures 2(c) and 2(d)). The differences between the nonlocal discrete regularization on $R G B$ color vectors (Figure 2(d)) or Chromaticity-Brightness features (Figure 2(e)) is now less evident (notice that the face of the goal-keeper is better restored with $\mathrm{CB}$ features). Even is it is visually evident that the nonlocal version of the proposed parameterless discrete regularization provides better results, its has another interesting property. This is shown in Figure 3. Figure $3(\mathrm{a})$ is the classical Barbara image corrupted with Gaussian noise $(\sigma=15)$. Figures 3(b) and 3(c) present the filtering results respectively with the weight functions $g_{2}$ and $g_{3}$ (i.e. local versus nonlocal). The differences are not so marked, but if we more precisely study their differences, the filtering with the $g_{3}$ weight function performs much more better. Indeed, it better preserves texture. This effect can be seen in Figures 3(e) and 3(f) where cropped and zoomed portions of Figures 3(b) and 3(c) are shown. Figure 3(d) provides the same cropped and zoomed part for the original corrupted image depicted in Figure 3(a). This texture preservation effect is due to the nature of the nonlocal weights as it was proposed in the original nonlocal means filter (see in $[13,15]$ ). 


\section{Conclusion}

In this paper, we have considered a discrete regularization framework based on graph differential geometry. The discrete regularization is based on the pLaplacian and leads to a family of linear and nonlinear iterative filters. Moreover, a parameterless version of the considered discrete regularization is also proposed. This enables to automatically estimate all the required parameters. Then, the obtained parameterless discrete regularization can be applied to a wide range of color image filtering applications with a proper choice of the weight function. The abilities of the proposed parameterless discrete regularization have been illustrated on sample examples. Future works will concern the adaptation of the proposed framework for image inpainting purposes.

\section{References}

1. Aubert, G., Kornprobst, P.: Mathematical Problems in Image Processing. Springer-Verlag (2002)

2. Chan, T., Shen, J.: Image Processing and Analysis - Variational, PDE, wavelet, and stochastic methods. SIAM (2005)

3. Tang, B., Sapiro, G., Caselles, V.: Color image enhancement via chromaticity diffusion. IEEE Transactions on Image Processing 10 (2001) 701-707

4. Tschumperlé, D., Deriche, R.: Vector-valued image regularization with PDEs: A common framework for different applications. IEEE Transactions on Pattern Analysis and Machine Intelligence 17(4) (2005) 506-517

5. Weickert, J.: Coherence-enhancing diffusion of colour images. Image Vision Comput. 17(3-4) (1999) 201-212

6. Zhou, D., Scholkopf, B.: A regularization framework for learning from graph data. In: ICML Workshop on Statistical Relational Learning and Its Connections to Other Fields. (2004) 132-137

7. Lezoray, O., Bougleux, S., Elmoataz, A.: Graph regularization for color image processing. Computer Vision and Image Understading (2007) in press

8. Bensoussan, A., Menaldi, J.L.: Difference equations on weighted graphs. Journal of Convex Analysis 12 (2005) 13-44

9. Diestel, R.: Graph Theory. Volume 173. Springer-Verlag (2005)

10. Chan, T., Osher, S., Shen, J.: The digital TV filter and nonlinear denoising. IEEE Transactions on on Image Processing 10 (2001) 231-241

11. Chambolle, A., Lions, P.L.: Image recovery via total variation minimization and related problems. Numerische Mathematik 76(2) (1997) 167-188

12. Chan, T., Kang, S., Shen, J.: Total variation denoising and enhancement of color images based on the CB and HSV color models. J. of Visual Communication and Image Representation 12 (2001) 422-435

13. Buades, A., Coll, B., Morel, J.: A non local algorithm for image denoising. In: IEEE Int. Conf. on Computer Vision and Pattern Recognition. Volume 2. (2005) $60-65$

14. Brook, A., Kimmel, R., Sochen, N.: Variational restoration and edge detection for color images. Journal of Mathematical Imaging and Vision 18 (2003) 247-268

15. Buades, A., Coll, B., Morel, J.: A review of image denoising algorithms, with a new one. Multiscale Modeling and Simulation (SIAM interdisciplinary journal) 4(2) (2005) 490-530 


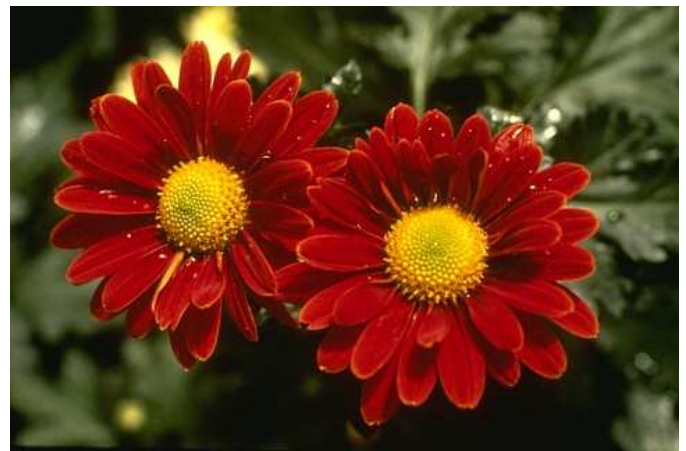

(a) Original image.

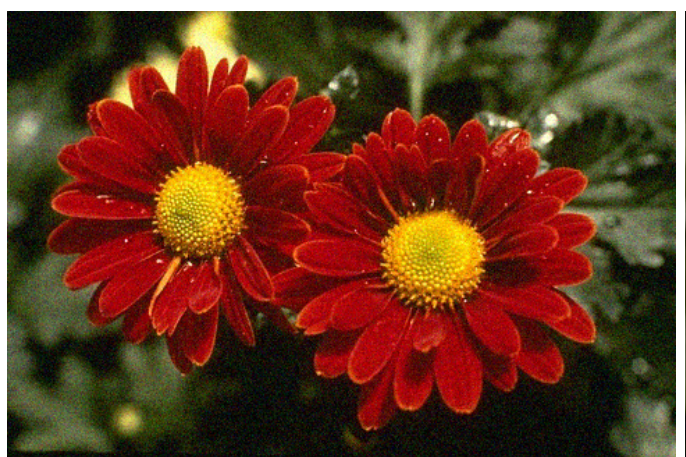

(c) Image distorted by Gaussian noise $(\sigma=15)$.

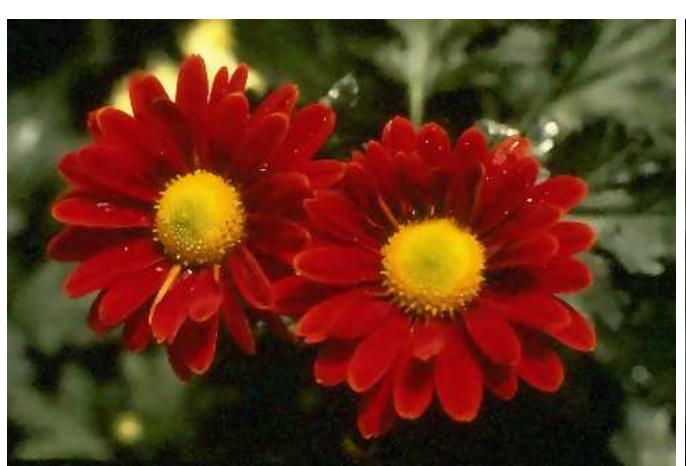

(e) Restoration of Gaussian noise with $g_{2}$.

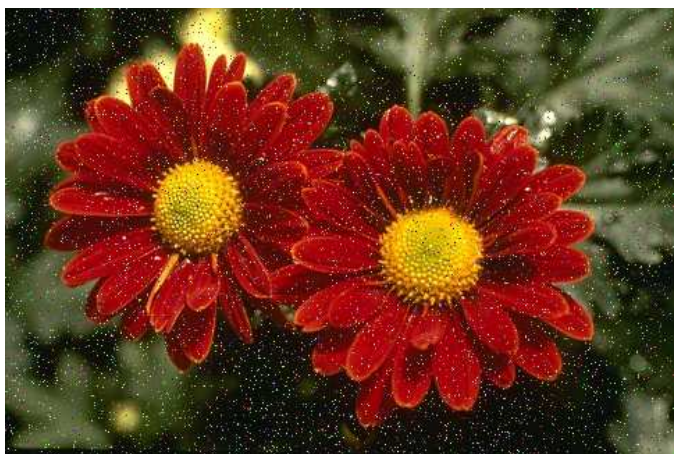

(b) Image distorted by $15 \%$ impulse noise.

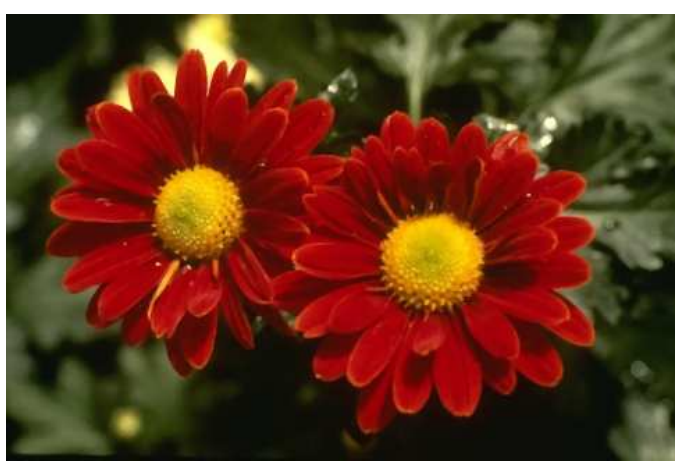

(d) Restoration of impulse noise with $g_{1}$.

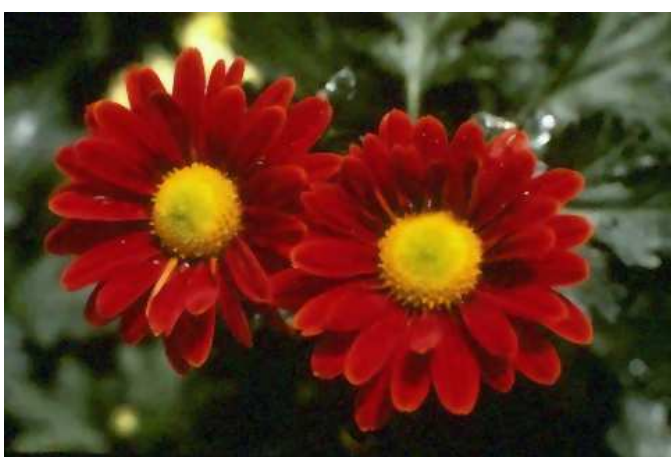

(f) Restoration of Gaussian noise with $g_{2}$ on $\mathrm{CB}$ features

Fig. 1. Illustrative examples of the filtering efficiency for two types of noise corruption and different edge weights functions. 


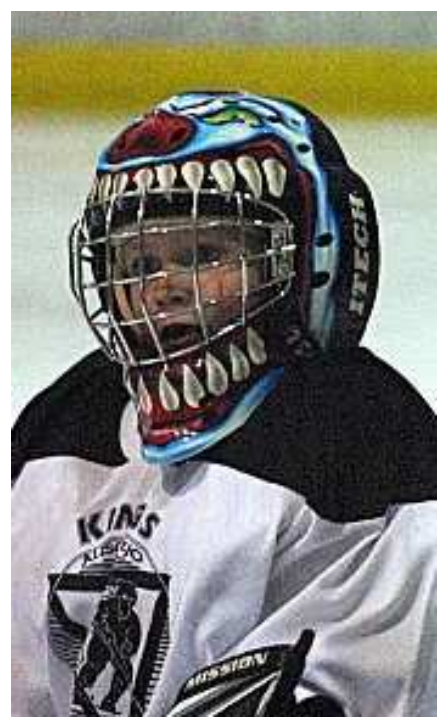

(a) Original noisy image.

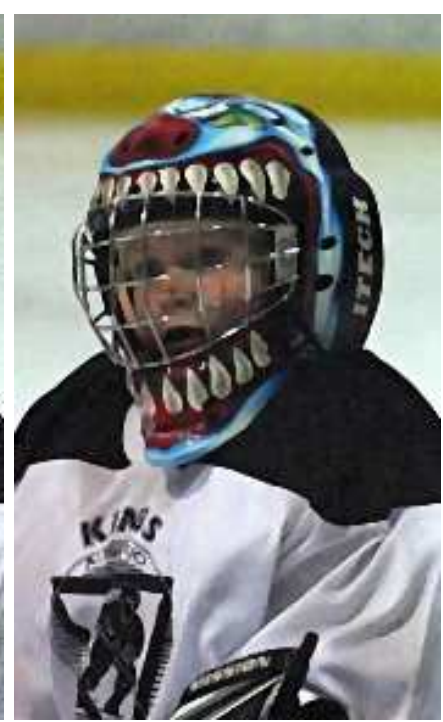

(b) Restored image with $g_{1}$.

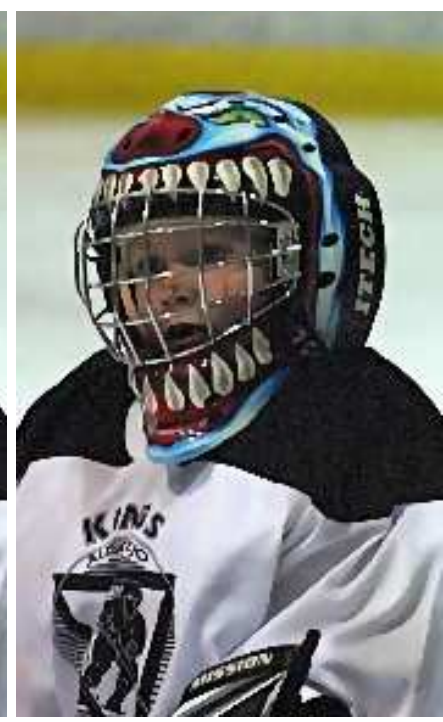

(c) Restored image with $g_{2}$.

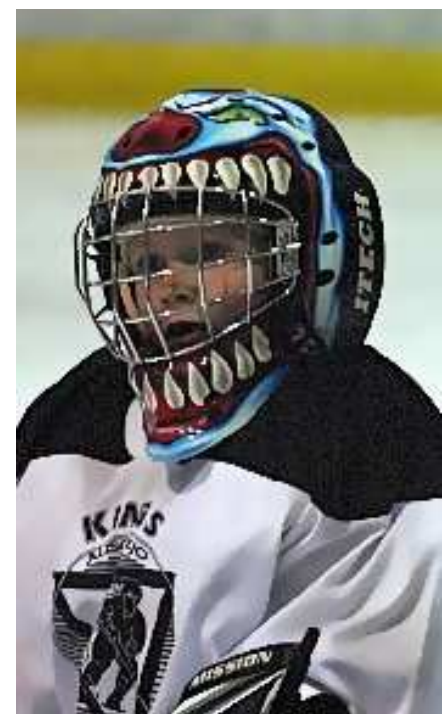

(d) Restored image with $g_{3}$.

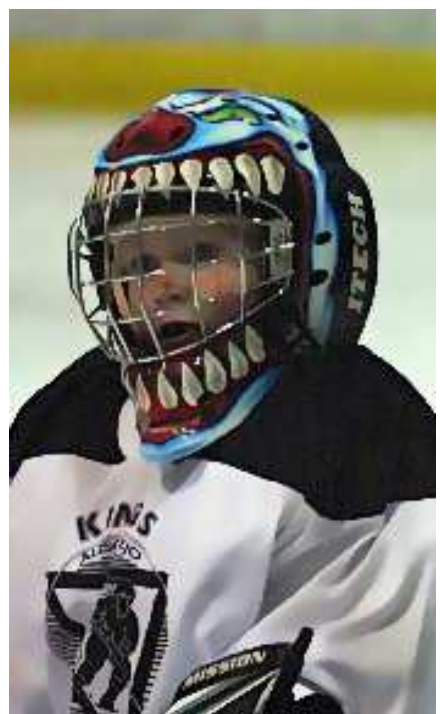

(e) Restored image with $g_{3}$ on CB features.

Fig. 2. Illustrative examples of the filtering efficiency on a real image corrupted by noise. 


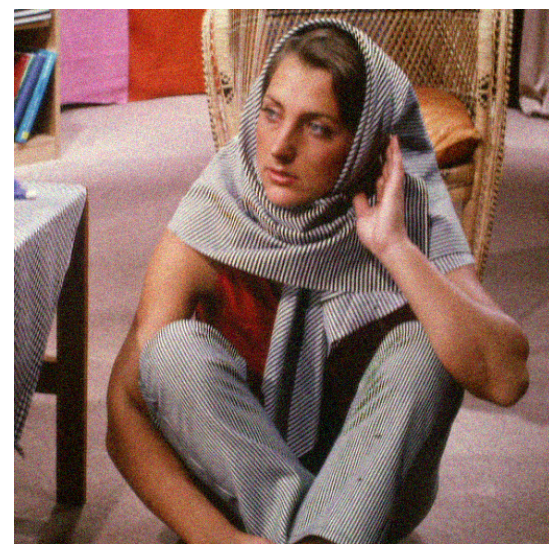

(a) Image with Gaussian noise $(\sigma=$ 15).

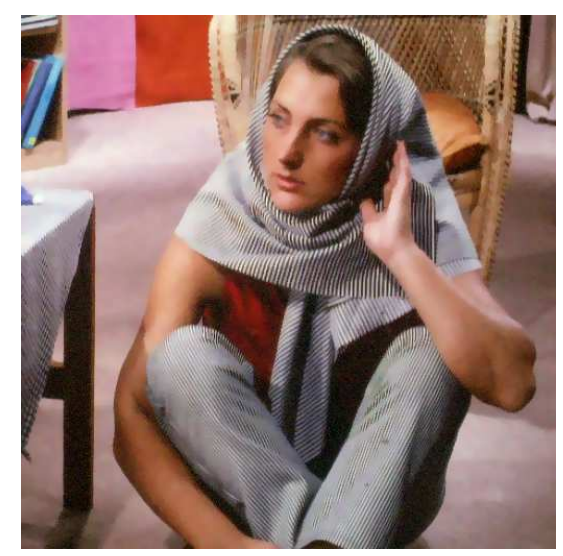

(c) Restored image with $g_{3}$.

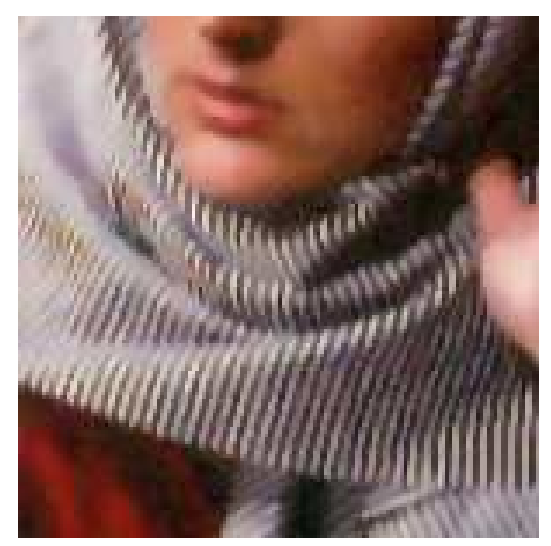

(e) Part of Figure 3(b).

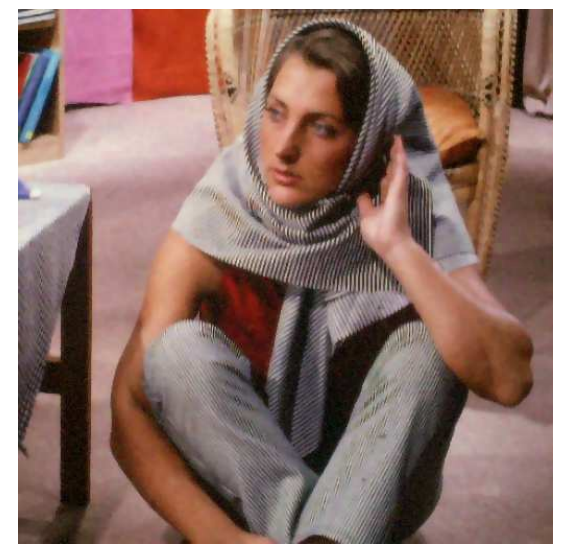

(b) Restored image with $g_{2}$.

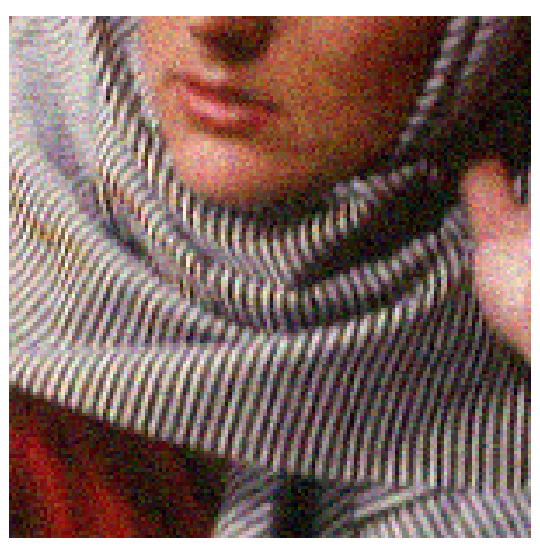

(d) Part of Figure 3(a).

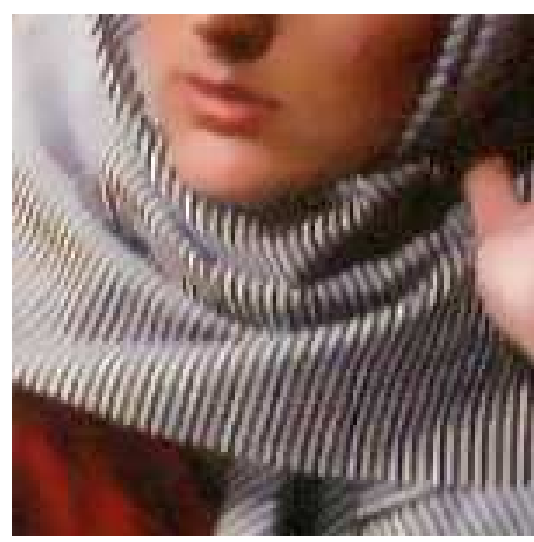

(f) Part of Figure 3(c).

Fig. 3. Illustrative examples of the differences between local and nonlocal weight functions. 